\title{
Measurement of the CKM Angles at BaBar and Belle.
}

\author{
Nick Barlow \\ University of Manchester - School of Physics and Astronomy \\ Oxford Road, Manchester - United Kingdom
}

\begin{abstract}
The primary goal of the BaBar and Belle experiments is to overconstrain the CKM Unitarity Triangle. Measurements of the angles of this triangle, known as $\beta, \alpha$, and $\gamma$ (or $\phi_{1}, \phi_{2}$, and $\phi_{3}$ ) give insight into the Standard Model description of $C P$ violation in the quark sector. BaBar and Belle have recorded almost $1 \mathrm{ab}^{-1}$ combined, and have measured $\beta$ to high precision. Measurements of $\alpha$ and $\gamma$ are less precise at present, but both experiments are rapidly accumulating data and developing new analysis techniques, and measurements of these angles will continue to provide useful constraints on the Standard Model description of $C P$ violation in the years to come.
\end{abstract}

\section{Introduction}

The BaBar and Belle experiments are both based at asymmetric-energy $e^{+} e^{-}$colliders operating at the $\Upsilon(4 S)$ resonance. Pairs of $B$ mesons are produced in a coherent state, so that if one $B$ decays into a flavour eigenstate at time $t=0$, the other $B$ must be the opposite flavour at that time. The asymmetric beam energies result in the $B$ mesons being boosted with respect to the laboratory frame, such that the decay distance (and hence the time difference $\Delta t$ ) between the two $B$ decays is measurable. If a $B$ decays into a final state $f_{C P}$ that is accessible from both $B^{0}$ and $\overline{B^{0}}$, interference between the situation where this $B$ decays directly into $f_{C P}$ and where it mixed into the opposite flavour before decaying can give rise to time-dependent $C P$ violation. By fully reconstructing the $B$ decay into $f_{C P}$, "tagging" the flavour of the other $B$, and measuring the time difference $\Delta t$ between their decays, we measure the time-dependend asymmetry $a_{f_{C P}}(\Delta t)$, which can be expressed as a sum of sine and cosine terms:

$$
\begin{aligned}
a_{f_{C P}}(\Delta t) & =\frac{\Gamma\left(B^{0}(\Delta t) \rightarrow f_{C P}\right)-\Gamma\left(\overline{B^{0}}(\Delta t) \rightarrow f_{C P}\right)}{\Gamma\left(B^{0}(\Delta t) \rightarrow f_{C P}\right)+\Gamma\left(\overline{B^{0}}(\Delta t) \rightarrow f_{C P}\right)} \\
& =S_{C P} \sin \left(\Delta m_{B} \Delta t\right)+C_{C P} \cos \left(\Delta m_{B} \Delta t\right),
\end{aligned}
$$

where $\Delta m_{B}$ is the mass difference between the two mass eigenstates of the neutral $B$ system. For decays with no direct $C P$ violation (i.e. $\Gamma(B \rightarrow f)=\Gamma(\bar{B} \rightarrow f)$ ), the coefficient $C_{C P}$ of the cosine term is expected to be zero, and the asymmetry will oscillate sinusoidally with an amplitude $S_{C P}$ that can often be directly related to one of the angles of the Unitarity Triangle.

\section{Measuring the angle $\beta\left(\phi_{1}\right)$}

\section{$2.1 \quad b \rightarrow c \bar{c} s$ decays}

The "golden channel" for measuring $\beta$ at the $B$ factories is $B \rightarrow J / \psi K_{S}^{0}$. No direct $C P$ violation is expected in this decay channel, as the tree diagram and the leading penguin diagram have the same weak phase. This means that the coefficient $C_{C P}$ of the cosine term in Eq. 1 is expected to be zero, while $S_{C P}=\sin (2 \beta)$. In addition, the branching 
fraction is relatively large $\left(\mathcal{O}\left(10^{-3}\right)\right.$, and the decay of a $J / \psi$ into two leptons gives a clear experimental signature. New results in this decay channel were announced in summer 2006 by both BaBar, based on $384 \mathrm{M} B \bar{B}$ events [2], and Belle, based on $535 \mathrm{M} B \bar{B}$ events [3]. BaBar also include the decay channels $\psi(2 S) K_{S}^{0}, \eta_{c} K_{S}^{0}$ and $\chi_{c 1} K_{S}^{0}$ that have $C P$ eigenvalue -1 , but Belle omits these in order to obtain a higher purity sample. Both experiments also use the decay channel $B \rightarrow J / \psi K_{L}^{0}$, which has the opposite $C P$ eigenvalue. This sample is much less pure due to the difficulty of reconstructing the $K_{L}^{0}$, but is nonetheless useful in reducing the statistical error on $\sin (2 \beta)$. BaBar measure $\sin (2 \beta)=0.714 \pm 0.032 \pm 0.018$, while Belle obtain $\sin (2 \beta)=0.642 \pm 0.031 \pm 0.017$.

\subsection{Penguin-dominated decays}

It is also possible to measure $\sin (2 \beta)$ using decays with $b \rightarrow q \bar{q} s$ transitions, where $q$ is a down-type quark $(s$ or $d)$. These involve flavourchanging-neutral currents, which are only possible at loop level in the Standard Model, and in some cases such as $B \rightarrow \phi K^{0}$, the decay is expected to be completely penguin-dominated, resulting in negligible direct $C P$ violation (which might otherwise arise through the interference between tree and penguin amplitudes). It is interesting to compare the value of $\sin (2 \beta)$ obtained with these channels with that obtained from $b \rightarrow c \bar{c} s$, as any deviation could be due to New Physics particles contributing to the loop. Recently, both BaBar and Belle observed statistically significant $C P$ violation in the decay channel $B \rightarrow \eta^{\prime} K^{0}$. With a dataset containing 384M $B \bar{B}$ events, BaBar [6] measure $S_{C P}=$ $0.58 \pm 0.10 \pm 0.03, C_{C P}=-0.16 \pm 0.07 \pm 0.03$. Belle [3] measure $S_{C P}=0.64 \pm 0.10 \pm 0.04$, $C_{C P}=0.01 \pm 0.07 \pm 0.05$ using $535 \mathrm{M} B \bar{B}$ events. Figure 1 is a plot from the Heavy Flavor Aver-

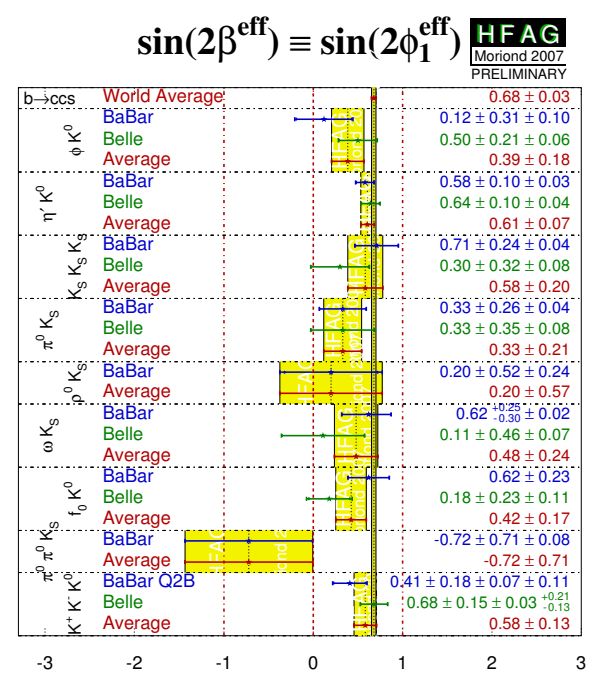
aging Group (HFAG) [7], showing a comparison of $S_{C P}$ measurements in penguin-dominated decays with the value measured in $B$ decays to charmonium. It can be seen that all the penguin modes tend to give lower values, and while this is not yet statistically significant, it will be extremely interesting to see if this discrepancy persists as BaBar and Belle accumulate more data.

\section{Measuring the angle $\alpha\left(\phi_{2}\right)$}

The angle $\alpha$ can be measured using $b \rightarrow u \bar{u} d$ decays, such as $B \rightarrow \pi^{+} \pi^{-}, B \rightarrow \rho^{+} \rho^{-}$ and $B \rightarrow \rho^{+} \pi^{-}$. However, for all these decay channels, it is expected that both tree and penguin diagrams will contribute, leading to possible direct $C P$ violation, such that the amplitude $S_{C P}$ of the time-dependent asymmetry oscillation is $\sin \left(2 \alpha_{e f f}\right)$ rather than $\sin (2 \alpha)$. Gronau and London [8] have outlined a technique for separating out the tree and 
penguin contributions using an isospin analysis based on the relative branching fractions for $B \rightarrow \pi^{+} \pi^{-}, B \rightarrow \pi^{ \pm} \pi^{0}$, and $B \rightarrow \pi^{0} \pi^{0}$ (this is also applicable to $\rho^{ \pm}$and $\rho^{0}$ ). However, some of these decay channels have small branching fractions and are experimentally hard to measure, so the uncertainty on the measured value of $\alpha$ is still largely due to the uncertainty on $\left(\alpha-\alpha_{e f f}\right)$.

For the decay channel $B \rightarrow \pi^{+} \pi^{-}$, Belle measure $S_{C P}=-0.61 \pm 0.10 \pm 0.04$ and $C_{C P}=$ $-0.55 \pm 0.08 \pm 0.05[9]$, using $535 \mathrm{M} B \bar{B}$ events. BaBar measure $S_{C P}=-0.6 \pm 0.11 \pm 0.03$ and $C_{C P}=-0.21 \pm 0.09 \pm 0.02[10]$. There is still some discrepancy between the BaBar and Belle results for $C_{C P}$, though this is smaller than in previous publications from both collaborations.

The decay channel $B \rightarrow \rho^{+} \rho^{-}$has the additional complication that it is a pseudoscalarto-vector-vector decay, and so the final state could potentially be a mixture of $C P$-odd and $C P$-even eigenstates. However, it turns out that the decay is almost $100 \%$ longitudinally polarized. In addition, the measured ratio of branching fractions for $B \rightarrow \rho^{0} \rho^{0}$ and $B \rightarrow$ $\rho^{ \pm} \rho^{0}[11]$ indicates that the fractional contribution to the decay amplitude from penguin diagrams is smaller than for $B \rightarrow \pi^{+} \pi^{-}$. This makes $\rho^{+} \rho^{-}$the best single channel for measuring $\alpha$. With a dataset containing 535M $B \bar{B}$ events, Belle measures $S_{C P}=0.19 \pm$ $0.30 \pm 0.08$ [12], while BaBar measure $S_{C P}=-0.17 \pm 0.20_{-0.06}^{+0.05}$ [13] using $384 \mathrm{M} B \bar{B}$ pairs.

It is worth noting that all these measurements of $\sin \left(2 \alpha_{e f f}\right)$ give multiple possible solutions for the value of $\alpha$. The CKMFitter [14] and UTFit[15] collaborations have averaged the measurements from both experiments, for all decay channels, using frequentist and Bayesian techniques respectively. Both groups find one of the solutions is compatible with the Standard Model: CKMFitter obtain $\alpha=\left(93_{-9}^{+11}\right)^{\circ}$ and UTFit find $\alpha$ to be between $81^{\circ}$ and $105^{\circ}$ at the $95 \%$ C.L.

\section{Measuring the angle $\gamma\left(\phi_{3}\right)$}

The angle $\gamma$ is the phase of the CKM matrix element $V_{u b}$, and is experimentally the hardest to reach at the $B$ factories. It can be measured in $B^{ \pm} \rightarrow D K^{ \pm}$decays where the $D$ decays into a final state accessible to both $D^{0}$ and $\overline{D^{0}}$. Interference between the colour-allowed decay $B^{+} \rightarrow$ $\overline{D^{0}} K^{+}$and the colour suppressed decay $B^{+} \rightarrow$ $D^{0} K^{+}$(and likewise for $B^{-}$) gives rise to $C P$ violation, but unfortunately the ratio of coloursuppressed to colour-allowed branching fractions is small, and therefore the interference is hard to measure. Three techniques are used at the $B$ factories: the $G L W$ method [16] where the $D$ decays into a $C P$ eigenstate; the $A D S$ method [17], where the $D$ decays into $K \pi$; and the $G G S Z$ method [18], using the Dalitz plot of the $D$ decay into $K_{S}^{0} \pi^{+} \pi^{-}$. With the GGSZ technique,

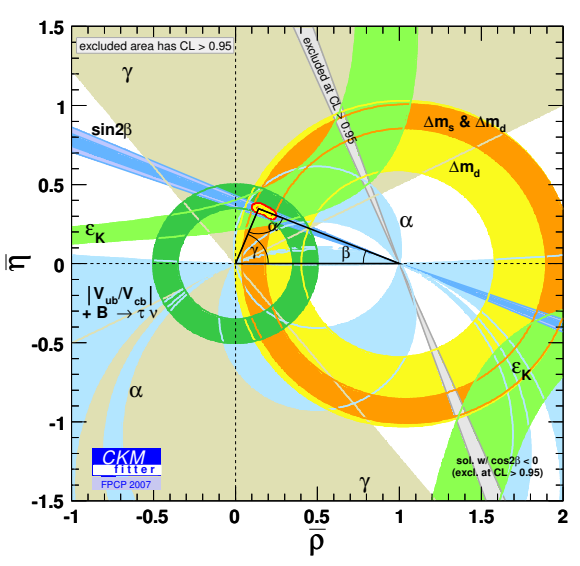

Figure 2: Constraints on the position of the apex of the Unitarity Triangle from all measurements. BaBar measures $\gamma=(92 \pm 42 \pm 10 \pm 13)^{\circ}$ [19] with $347 \mathrm{M} B \bar{B}$ events. Belle uses a sample of $386 \mathrm{M} B \bar{B}$ events to obtain $\gamma=\left(53_{-18}^{+15} \pm 13 \pm 9\right)^{\circ}$ [20]. In both cases, the third error is due to the uncertainty in the Dalitz model. 


\section{Conclusions}

Figure 2 shows a fit from the CKMFitter collaboration [14] illustrating the current constraints on the position of the apex of the CKM Unitarity Triangle from measurements of its sides and angles. At present, all measurements are compatible with each other and with the Standard Model, and the measurement of $\beta$ provides one of the strongest constraints. The uncertainties on the angles $\beta, \alpha$, and $\gamma$ are approximately $1^{\circ}, 10^{\circ}$ and $35^{\circ}$ respectively. In the coming years, as BaBar and Belle accumulate more data, the uncertainties on $\gamma$ and in particular on $\alpha$ will reduce substantially, and will provide an excellent test of the Standard Model description of $C P$ violation.

\section{Bibliography}

\section{References}

[1] Slides: http://indico. cern.ch/contributionDisplay . py? contribId=115\&sessionId=9\&conf $I d=9499$

[2] B. Aubert et al., arXiv:hep-ex/0703021, submitted to Phys. Rev. Lett.

[3] K. F. Chen et al., Phys. Rev. Lett. 98031892 (2007).

[4] S. Fratina et al., Phys. Rev. Lett. 98221802 (2007).

[5] B. Aubert et al., arXiv:0705.1190, submitted to Phys. Rev. Lett.

[6] B. Aubert et al., Phys. Rev. Lett. 98031801 (2007).

[7] http://www.slac.stanford.edu/xorg/hfag

[8] M. Gronau and D. London, Phys. Rev. Lett. 653381 (1990).

[9] H. Ishino et al., Phys. Rev. Lett 98211801 (2007).

[10] B. Aubert et al., Phys. Rev. Lett. 99021603 (2007).

[11] B. Aubert et al., Phys. Rev. Lett. 97261801 (2006) B. Aubert et al., Phys. Rev. Lett. 98111801 (2007).

[12] K. Abe et al., Phys. Rev. D76 011104 (2007).

[13] B. Aubert et al., arXiv:0705.2157, submitted to Phys. Rev. D.

[14] http://ckmfitter.in2p3.fr/

[15] http://utfit.roma1.infn.it/

[16] M. Gronau and D. London, Phys. Lett. B253 483 (1991). M. Gronau and D. Wyler, Phys. Lett. B265 172 (1991).

[17] D. Atwood, I. Dunietz and A. Soni, Phys. Rev. D63 036005 (2001).

[18] A. Giri, Y. Grossman, A. Soffer and J. Zupan, Phys. Rev. D68 054018 (2003).

[19] B. Aubert et at., arXiv:hep-ex/0707104.

[20] A. Poluektov et al., Phys. Rev. D73 112009 (2006). 